\title{
ProRaris: Menschen mit seltenen Krankheiten eine Stimme geben
}

\author{
Jacqueline de Sáa, Hermann Amstad ${ }^{b}$ \\ ${ }^{a}$ Dr., Geschäftsführerin ProRaris, Bern; ${ }^{b}$ Dr., amstad-kor, Basel
}

\begin{abstract}
Am 29. Februar 2020 feiert ProRaris in Genf das 10-Jahr-Jubiläum. Der gemeinnützige Verein dient als Sprachrohr von Menschen mit seltenen Krankheiten und vertritt deren Anliegen gegenüber den Behörden, aber auch in der Öffentlichkeit. Angesichts der weiterhin zahlreichen Aufgaben möchte ProRaris seine Basis erweitern; mit einer Sympathiemitgliedschaft kann man die Anliegen von ProRaris unterstützen.
\end{abstract}

Eine Krankheit gilt dann als selten, wenn sie in der Bevölkerung weniger als 1 von 2000 Personen betrifft. Da es aber 6000 bis 8000 seltene Krankheiten gibt, sind in der Schweiz mehr als eine halbe Million Menschen davon betroffen. Die Grössenordnung ist vergleichbar mit Diabetes, einer Krankheit, die deutlich

In der Schweiz sind mehr als eine halbe Million Menschen von einer seltenen Krankheit betroffen.

stärker im öffentlichen Bewusstsein verankert ist. Dennoch fand das Thema «seltene Krankheiten» in der Schweiz lange Zeit nur wenig Beachtung.

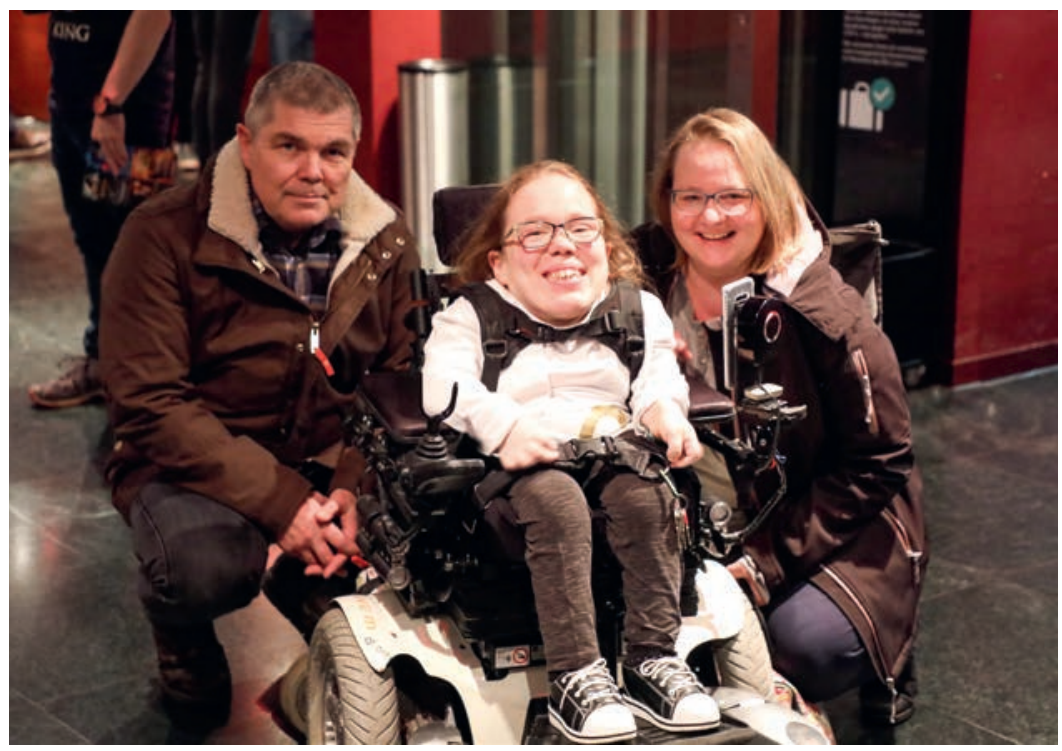

Dr. Alfred Wiesbauer, Vizepräsident von ProRaris, mit seiner Frau Christine und seiner Tochter Sophie, die an Mukopolysaccharidose IVa (Morbus Morquio) leidet.
2009 startete die Europäische Union eine Initiative zur Verbesserung der Situation von Menschen mit seltenen Erkrankungen. Dieser Impuls strahlte auch in die Schweiz aus. Im Juni 2010 wurde «ProRaris Allianz Seltener Krankheiten - Schweiz» als gemeinnütziger Verein gegründet, dies mit dem Ziel, die Patientenorganisationen im Bereich der seltenen Krankheiten $\mathrm{zu}$ vereinigen und ihnen so eine starke Stimme zu geben. Die Allianz vereinigt heute rund 60 Patientenorganisationen und Stiftungen im Bereich seltene Krankheiten.

Ein weiterer Meilenstein war die Gründung der Tochterorganisation UniRares im September 2018. Schon seit den Anfängen von ProRaris war es ein Anliegen, auch den Patientinnen und Patienten, welche über keine krankheitsspezifische Organisation verfügen, Gehör zu verschaffen. UniRares vereinigt die «isolierten Patienten» und auch solche ohne Diagnose. ProRaris unterstützt diese junge Vereinigung administrativ und finanziell.

\section{Am 29. Februar ist «Rare Disease Day»}

Eurordis, die Europäische Organisation für Seltene Krankheiten, organisiert seit 2007 jährlich einen «Rare Disease Day"; symbolisch wurde dieser Tag auf den 29. Februar gelegt. Im Februar 2011 organisierte ProRaris erstmals auch in der Schweiz einen "Tag der Seltenen Krankheiten». Kurz zuvor hatte das Bundesgericht seinen umstrittenen Entscheid zur Vergütung von Myozym, einer "Orphan Drug», gefällt. Das Gericht stellte dabei fest, dass die Krankenkasse das Medikament zur Behandlung der seltenen Krankheit Morbus Pompe nicht bezahlen müsse, weil das Kosten- 
Nutzen-Verhältnis nicht adäquat sei. Dieses Urteil stand dann im Zentrum des Anlasses, der mit einer Teilnehmerzahl von über 400 Personen ein grosser Erfolg war.

Seither organisiert ProRaris jährlich einen Tag der seltenen Krankheiten. Der Tag bietet Patientinnen und Patienten, Angehörigen, Patientenorganisationen, Gesundheitsfachleuten, Forschenden, aber auch Medienleuten sowie der breiten Öffentlichkeit die Möglichkeit, sich bei Vorträgen und Gesprächen am runden Tisch, aber auch im direkten Kontakt auszutauschen. Zum 10-jährigen Jubiläum kehrt ProRaris an den Ort der Anfänge zurück: Am 29. Februar 2020 findet am Unispital Genf der 10. Tag der Seltenen Krankheiten in der Schweiz statt. Das vielfältige Programm bietet einen Rückblick auf die Anfänge der Organisation, eine Diskussionsrunde mit den Mitgliederorganisationen und einen Ausblick auf die zukünftige Versorgung der Betroffenen. Der Anlass ist öffentlich.

\section{Das «Nationale Konzept Seltene Krank- heiten": ein erster Erfolg}

Seit der Gründung hat ProRaris bereits einiges erreicht. Im Jahr 2014 verabschiedete der Bundesrat das

\section{Das Thema "seltene Krankheiten" wurde in den Lernzielkatalog der Humanmedizin aufgenom- men und ist künftig prüfungsrelevant.}

Nationale Konzept für Seltene Krankheiten und 2015 die dazugehörige Umsetzungsplanung. Mit dem Nationalen Konzept soll der gleichberechtigte Zugang zu Diagnose, Therapie und Versorgung für alle von seltenen Krankheiten betroffenen Menschen erreicht werden. Zudem sollen psychosoziale Dienstleistungen für Patientinnen und Patienten sowie ihre Angehörigen bekannter und leichter zugänglich gemacht werden. Einiges wurde im Rahmen des Nationalen Konzepts bereits umgesetzt. So wurde z.B. das Thema «seltene

Weitere Links zu seltenen Krankheiten

\footnotetext{
- UniversitätsSpital Zürich und Universitäts-Kinderspital Zürich: www.zentrumseltenekrankheiten.ch

- Netzwerk Rare Diseases Nordwest und Zentralschweiz: www.ukbb.ch/de/Rare-Diseases.php

- Universitätsspitäler Lausanne und Genf: www.info-maladies-rares.ch

- Malattie genetiche rare Ticino: www.malattiegeneticherare.ch

- Informationsportal: www.orpha.net

- Nationale Koordination Seltene Krankheiten:

www.kosekschweiz.ch
}

Krankheiten" in den Lernzielkatalog der Humanmedizin aufgenommen und ist künftig prüfungsrelevant. Auch die Arbeiten am Schweizer Register Seltene Krankheiten sind weit fortgeschritten. Zudem wurden in der Deutschschweiz, der Romandie und im Tessin Helplines eingerichtet, die auf das Thema spezialisiert sind.

Neben der Allianz, welche als Schlüsselpartnerin bezeichnet wurde, haben sich zahlreiche Akteure aus medizinischer Forschung und Praxis sowie aus den

\section{Im Juni 2017 wurde die Nationale Koordination} Seltene Krankheiten (kosek) gegründet.

Kantonen mit grossem Engagement an der Umsetzung beteiligt. Im Juni 2017 wurde die Nationale Koordination Seltene Krankheiten (kosek) gegründet. ProRaris ist Gründungsmitglied und engagiert sich seither mit den anderen Partnern für den Aufbau der Zentren für seltene Krankheiten, die allen betroffenen Patienten den Zugang zu medizinischen Dienstleistungen ermöglichen sollen. Auch die Arbeiten für die krankheitsspezifischen Referenzzentren sind im Gange.

\section{ProRaris braucht eine breitere Basis}

Trotz der Erfolge braucht es weitere Anstrengungen, damit die bisher aufgebauten Strukturen längerfristig Bestand haben und optimiert werden können. Dabei bleibt auch die Rolle von ProRaris weiterhin wichtig, um die Umsetzung der Massnahmen im Sinne der Patienten und Patientinnen zu sichern. Die grösste Herausforderung sind dabei die Finanzen. Wegen fehlender rechtlicher Grundlagen gilt, wie in der Umsetzungsplanung festgehalten, dass jeder Akteur die nötigen Mittel für die von ihm unternommenen Massnahmen selbst bereitstellt. Um ihre Aktivitäten zu finanzieren, ist ProRaris daher auf Spendengelder angewiesen (IBAN CH22 00767000 E525 2446 2, ProRaris, Vuarrens). Eine weitere Möglichkeit, ProRaris zu unterstützen, ist die Sympathiemitgliedschaft. Diese steht juristischen oder natürlichen Personen offen, welche die Bedingungen für eine Aktivmitgliedschaft nicht erfüllen, sich aber am Kampf gegen seltene Krankheiten beteiligen möchten. Eine Anmeldung ist jederzeit möglich auf der Website von ProRaris (www.proraris. ch) unter «Mitglieder».

Lesen Sie zum Thema «seltene Krankheiten» das Interview mit Prof. Matthias Baumgartner auf den Seiten 71-72.

Bildnachweis

(c) Alfred Wiesbauer 